\title{
Measurements of event shapes and jet substructure with ATLAS Run 2 data
}

\author{
M. LeBlanc, ${ }^{a, *}$ on behalf of the ATLAS collaboration \\ ${ }^{a}$ University of Arizona, \\ 1118 E. Fourth Street, Tucson, USA \\ E-mail: matt. leblanc@cern.ch
}

In order to achieve the highest levels of precision at the Large Hadron Collider, a detailed understanding of the strong interaction is required. Three recent measurements made by the ATLAS collaboration in $\sqrt{s}=13 \mathrm{TeV} p p$ collisions are reported, which are sensitive to different aspects of perturbative and non-perturbative quantum chromodynamics. These results include a measurement of hadronic event shapes in multijet final states with large momentum transfer, a measurement of jet substructure quantities with jets groomed using the Soft Drop algorithm, and a measurement of the Lund jet plane using charged particle tracks inside of jets. These measurements are corrected for acceptance and detector effects, and are compared to state-of-the-art Monte Carlo models and analytical calculations. The measured data have been made publicly available for use in future studies.

The Eighth Annual Conference on Large Hadron Collider Physics-LHCP2020

25-30 May, 2020

online

${ }^{*}$ Speaker 


\section{Introduction}

In order to achieve the highest levels of precision at the Large Hadron Collider, a detailed understanding of the strong interaction is required. Three recent measurements made by the ATLAS collaboration [1] in $\sqrt{s}=13 \mathrm{TeV}$ proton-proton collisions are reported, which are sensitive to different aspects of perturbative and non-perturbative quantum chromodynamics. These measurements are corrected for acceptance and detector effects, and are compared to state-of-the-art Monte Carlo (MC) models [2] and available analytical calculations.

\section{Measurement of hadronic event shapes in multijet final states}

Event shapes are a family of observables that are used to characterise the flow of energy in collider events. Measurements of event shapes have been used to probe fundamental properties of QCD [39], to tune MC models [10] and to search for physics beyond the Standard Model (SM) [11]. ATLAS has performed new measurements of several event shape observables $[12,13]$, revisiting this topic for the first time since early in Run 1 .

The event shapes measured include the transverse thrust $T_{\perp}$ and its minor component $T_{m}[14$, 15]; the aplanarity $A$ and sphericity $S$, including the transverse sphericity projection $S_{\perp}$ [16, 17]; and the $C$ and $D$ event shapes, which respectively vanish for two-jet and planar events. The large Run 2 dataset [18], advances in jet reconstruction performance [19] and MC models [2] allow these measurements to be binned in the jet multiplicity and the sum of the leading and subleading jet $p_{\mathrm{T}}$. The measurements are normalised to the inclusive cross section of events with at least two jets.

The achieved precision tends to be limited at low jet multiplicity by differences in predictions from the various MC models studied in the context of the unfolding procedure (e.g. Figure 1(a)), and at high jet multiplicity by the jet energy scale (e.g. Figure 1(b)). The dominant component of the latter uncertainty is due to differences in the modelling of the gluon-jet response between Pythia 8 and Herwig++. The precision of the measurements depends on the fiducial region, but generally ranges between $1 \%-10 \%$. Several MC models are compared to the measured data. The simulation describes the main features of the measurements, but agreement degrades in regions with large non-perturbative effects and in events with high jet multiplicity.

\section{Measurement of soft-drop jet observables}

Jet substructure (JSS) presents new opportunities to learn about the strong interaction at high energy scales. Of particular interest in this context is the substructure of jets which have been groomed with the soft-drop (SD) algorithm [20, 21], which removes soft- and wide-angled radiation in a way that allows the groomed JSS distributions to be precisely described analytically. ATLAS has published a measurement of several JSS observables which characterise the hard splitting within a jet following SD grooming [22, 23], expanding on a prior measurement of the SD jet mass [24].

The SD algorithm proceeds by reclustering the constituents of a jet using the Cambridge-Aachen (C/A) jet clustering algorithm [25, 26], then iterating through the angularly-ordered clustering history starting from the widest-angled splitting. At each step, the splitting between subjets $j_{1}$ and 
$j_{2}$, respectively the harder and softer branches of the clustering, is subjected to the SD condition:

$$
\frac{\min \left(p_{\mathrm{T}}^{j_{1}}, p_{\mathrm{T}}^{j_{2}}\right)}{p_{\mathrm{T}}^{j_{1}}+p_{\mathrm{T}}^{j_{2}}}>z_{\text {cut }}\left(\frac{\Delta R\left(j_{1}, j_{2}\right)}{R}\right)^{\beta},
$$

where $R$ is the ungroomed jet radius (here, $R=0.8$ ), and $z_{\text {cut }}$ and $\beta$ are parameters which control how much radiation is removed. If the splitting fails this condition, the softer subjet $j_{2}$ is discarded and grooming proceeds using $j_{1}$. If the inequality is satisfied, then the SD procedure terminates. In this measurement, dijet events with a leading jet $p_{\mathrm{T}}>300 \mathrm{GeV}$ are selected. The SD parameter $z_{\text {cut }}$ is fixed at 0.1 , and values of $\beta \in\{0,1,2\}$ are studied.

The properties of the hard splitting that stops the grooming procedure are studied, measuring the groomed jet mass $m$, the $p_{\mathrm{T}}$ balance of the two primary subjets $z_{g}=j_{2} / j_{1}$ and their opening angle, $r_{g}=\Delta R\left(j_{1}, j_{2}\right)$. These quantities are measured using calorimeter- and inner-detector based signals. The measured distributions are decomposed into quark- and gluon-like components using information from simulation. The inner-detector based measurements are more precise overall, but omit the neutral component of jet fragmentation. The calorimeter-based measurements contain information about all particles, and so these $m$ and $r_{g}$ measurements may be compared to several cutting-edge analytical predictions [27-33]. Good agreement between the experimental data and these predictions is observed in regions where resummation effects are largest $\left(\Lambda_{\mathrm{QCD}} / p_{\mathrm{T}} \leq m / p_{\mathrm{T}}<\right.$ $\left.z_{\text {cut }}\right)$. The accuracy of the descriptions deteriorates particularly as non-perturbative effects become more relevant. The overall precision is generally limited due to differences in the results obtained by unfolding with different MC models (Figure 1(c)).

\section{Measurement of the Lund jet plane using charged particles}

Rather than studying only the hardest perturbative splitting within a jet's formation, a recent phenomenological proposal [34] suggests an approach based on the framework of Lund diagrams [35] which can probe the entire jet clustering history. ATLAS has published a measurement of the Lund jet plane (LJP) [36, 37], using an inclusive sample of high- $p_{\mathrm{T}}$ jets in $139 \mathrm{fb}^{-1}$ of Run $2 p p$ collision data. Lund diagrams for individual $R=0.4$ jets are constructed by reclustering a jet's charged constituents with the C/A algorithm. The resultant angularly-ordered clustering sequence is then iteratively declustered; at each step, the relative momentum fraction $(z)$ and angle $(\Delta R)$ of the softer branch are taken as proxies for the kinematics of emissions from the hard-scatter parton. Dijet events with $R=0.4$ jet $p_{\mathrm{T}}>675 \mathrm{GeV}$ are selected in this measurement.

The LJP is the two-dimensional space spanned by $\ln (1 / z) v s . \ln (R / \Delta R)$. Different physical effects factorise naturally in this space. This factorisation allows a single measurement to probe JSS in a way which is simultaneously sensitive to e.g. different parton shower and hadronisation models, but also isolates sensitivity to each process within expected regions of the LJP distribution. The precision of this measurement is typically $\sim O(10 \%)$, limited by differences in the final result observed when unfolding the data using different MC models (Figure 1(d)). The measured data tend to disagree significantly with the predictions of several state-of-the-art MC models, indicating that this measurement could be a useful resource for future non-perturbative model-building and parton shower MC tuning efforts. Recently, an analytical prediction of the primary LJP density 
has been published [38], which agrees well with this measurement in regions of the LJP where non-perturbative effects are small.

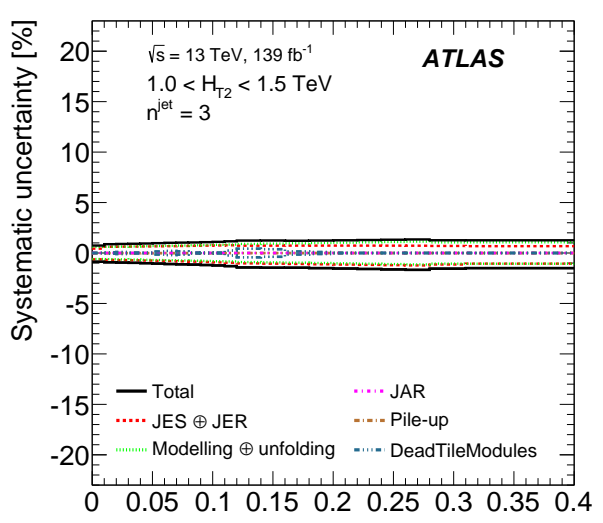

A

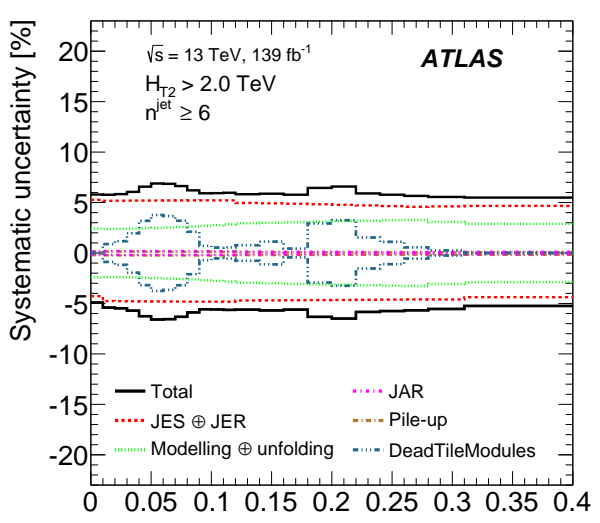

A (a)

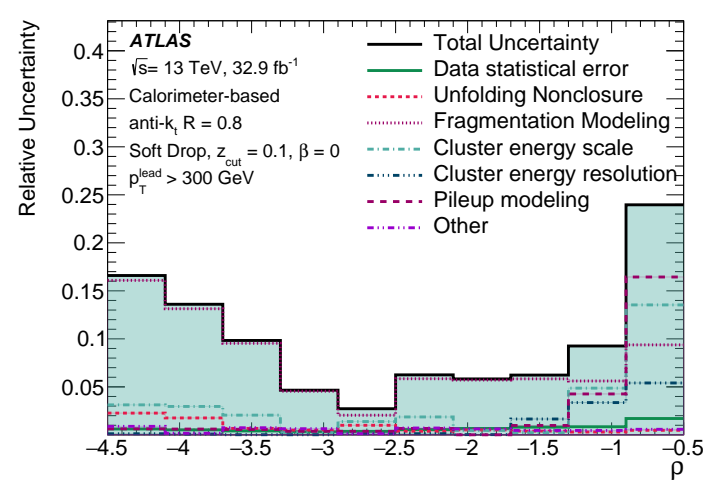

(c) (b)

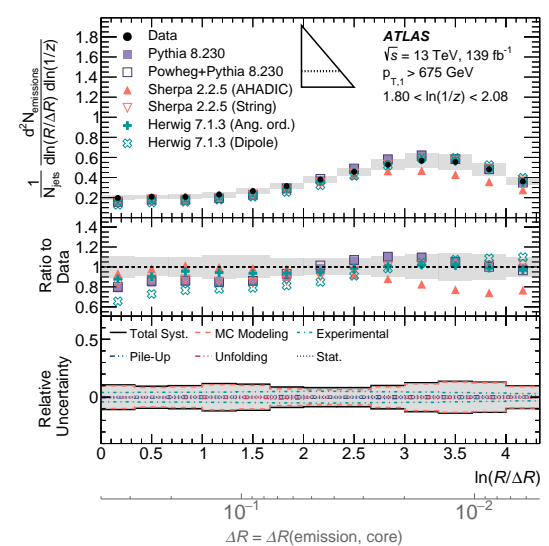

(d)

Figure 1: Selected figures from (a,b) Reference [12], (c) Reference [22] and (d) Reference [36]. In each case, the precision of these analyses is limited by differences between MC models. In cases where the experimental uncertainty due to the jet energy scale is large, the most relevant subcomponent of the uncertainty in these measurements is related to differences in the modelling of gluon jets between various standard MC generators.

\section{Concluding remarks}

ATLAS has a vibrant jet physics programme studying the dynamics of the strong interaction using both traditional differential cross-section measurements and cutting-edge JSS techniques. In each measurement presented here, and as highlighted in Figure 1, the precision is limited in at least some portion of the fiducial space by disagreements between MC models. The measured data have been made publicly available for use in future studies [23, 37], in order to help facilitate the maximum possible precision of future physics analyses performed at the LHC. 


\section{References}

[1] ATLAS Collaboration, The ATLAS Experiment at the CERN Large Hadron Collider, JINST 3 (2008) S08003.

[2] ATLAS Collaboration, Multijet simulation for $13 \mathrm{TeV}$ ATLAS Analyses, ATL-PHYS-PUB-2019-017, 2019. https://cds.cern.ch/record/2672252.

[3] JADE Collaboration, Observation of planar three-jet events in $e+e-$ annihilation and evidence for gluon bremsstrahlung, Physics Letters B 91 (1980) 142.

[4] S. Kluth, P.A. Movilla Fernandez, S. Bethke, C. Pahl and P. Pfeifenschneider, A Measurement of the QCD color factors using event shape distributions at $\sqrt{s}=14 \mathrm{GeV}$ to $189 \mathrm{GeV}$, Eur. Phys. J. C 21 (2001) 199, hep-ex/0012044.

[5] OPAL Collaboration, Determination of $\alpha_{s}$ using OPAL hadronic event shapes at $\sqrt{s}=91-209 \mathrm{GeV}$ and resummed NNLO calculations, Eur. Phys. J. C 71 (2011) 1733, 1101.1470 [hep-ex].

[6] S. Kluth, $\alpha_{s}\left(M\left(Z^{0}\right)\right)$ from Jade Event Shapes, in 44th Rencontres de Moriond on QCD and High Energy Interactions, pp. 295-298, 2009, 0905 . 4891 [hep-ex].

[7] H1 and ZEUS Collaborations, alpha(s) determinations from jets and scaling violations at HERA, in 26th International Symposium on Physics in Collision (PIC 2006), 2006, hep-ex/0610040.

[8] OPAL Collaboration, Measurement of $\alpha_{s}$ with radiative hadronic events, Eur. Phys. J. C 53 (2008) 21, 0902 . 1128 [hep-ex].

[9] Particle Data Group collaboration, M. Tanabashi et al., Review of Particle Physics, Phys. Rev. D 98 (2018) 030001.

[10] P. Skands, S. Carrazza and J. Rojo, Tuning PYTHIA 8.1: the Monash 2013 Tune, Eur. Phys. J. C 74 (2014) 3024, 1404.5630 [hep-ph].

[11] L. A. Anchordoqui, D. C. Dai, H. Goldberg, G. Landsberg, G. Shaughnessy, D. Stojkovic and T. J. Weiler, Searching for the layered structure of space at the LHC, Phys. Rev. D 83 (2011) 114046, 1012 . 1870 [hep-ph].

[12] ATLAS Collaboration, Measurement of hadronic event shapes in multijet final states at $\sqrt{s}=13 \mathrm{TeV}$ with the ATLAS detector, 2007.12600 [hep-ex].

[13] ATLAS collaboration, Measurement of hadronic event shapes in multijet final states at $\sqrt{s}=13 \mathrm{TeV}$ with the ATLAS detector, 2020. https://doi.org/10.17182/hepdata.91998.

[14] S. Brandt, C. Peyrou, R. Sosnowski and A. Wroblewski, The principal axis of jets - an attempt to analyse high-energy collisions as two-body processes, Physics Letters 12 (1964) 57 . 
[15] E. Farhi, Quantum Chromodynamics Test for Jets, Phys. Rev. Lett. 39 (1977) 1587.

[16] G. Parisi, Superinclusive cross sections, Physics Letters B 74 (1978) 65 .

[17] J. F. Donoghue, F. E. Low and S.-Y. Pi, Tensor analysis of hadronic jets in quantum chromodynamics, Phys. Rev. D 20 (1979) 2759.

[18] ATLAS Collaboration, ATLAS data quality operations and performance for 2015-2018 data-taking, JINST 15 (2020) P04003, 1911.04632 [physics.ins-det].

[19] ATLAS Collaboration, Jet energy scale and resolution measured in proton-proton collisions at $\sqrt{s}=13 \mathrm{TeV}$ with the ATLAS detector, 2007.02645 [hep-ex].

[20] M. Dasgupta, A. Fregoso, S. Marzani and G. P. Salam, Towards an understanding of jet substructure, JHEP 09 (2013) 029, 1307.0007 [hep-ph].

[21] A. J. Larkoski, S. Marzani, G. Soyez and J. Thaler, Soft drop, JHEP 05 (2014) 146, 1402.2657 [hep-ph].

[22] ATLAS collaboration, Measurement of soft-drop jet observables in pp collisions with the ATLAS detector at $\sqrt{s}=13 \mathrm{TeV}$, Phys. Rev. D 101 (2020) 052007, 1912.09837 [hep-ex].

[23] ATLAS collaboration, Measurement of soft-drop jet observables in pp collisions with the ATLAS detector at $\sqrt{s}=13 \mathrm{TeV}, 2020$. https://doi.org/10.17182/hepdata.92073.

[24] ATLAS Collaboration, Measurement of the Soft-Drop Jet Mass in pp Collisions at $\sqrt{s}=13 \mathrm{TeV}$ with the ATLAS Detector, Phys. Rev. Lett. 121 (2018) 092001, 1711.08341 [hep-ex].

[25] Y. L. Dokshitzer, G. D. Leder, S. Moretti and B. R. Webber, Better jet clustering algorithms, JHEP 08 (1997) 001, hep-ph/9707323.

[26] M. Wobisch and T. Wengler, Hadronization corrections to jet cross-sections in deep inelastic scattering, in Workshop on Monte Carlo Generators for HERA Physics (Plenary Starting Meeting), pp. 270-279, 1998, hep-ph/9907280.

[27] S. Marzani, L. Schunk and G. Soyez, A study of jet mass distributions with grooming, JHEP 07 (2017) 132, 1704.02210 [hep-ph].

[28] S. Marzani, L. Schunk and G. Soyez, The jet mass distribution after Soft Drop, Eur. Phys. J. C 78 (2018) 96, 1712.05105 [hep-ph].

[29] C. Frye, A. J. Larkoski, M. D. Schwartz and K. Yan, Factorization for groomed jet substructure beyond the next-to-leading logarithm, JHEP 07 (2016) 064, 1603.09338 [hep-ph].

[30] C. Frye, A. J. Larkoski, M. D. Schwartz and K. Yan, Precision physics with pile-up insensitive observables, 1603.06375 [hep-ph]. 
[31] Z.-B. Kang, K. Lee, X. Liu and F. Ringer, Soft drop groomed jet angularities at the LHC, Phys. Lett. B 793 (2019) 41, 1811.06983 [hep-ph].

[32] Z.-B. Kang, K. Lee, X. Liu and F. Ringer, The groomed and ungroomed jet mass distribution for inclusive jet production at the LHC, JHEP 10 (2018) 137, 1803.03645 [hep-ph].

[33] Z.-B. Kang, K. Lee, X. Liu, D. Neill and F. Ringer, The soft drop groomed jet radius at NLL, JHEP 02 (2020) 054, 1908.01783 [hep-ph].

[34] F. A. Dreyer, G. P. Salam and G. Soyez, The Lund jet plane, JHEP 12 (2018) 064, 1807.04758 [hep-ph].

[35] B. Andersson, G. Gustafson, L. Lönnblad and U. Pettersson, Coherence effects in deep inelastic scattering, Z. Phys. C $\mathbf{4 3}$ (1989) 625.

[36] ATLAS collaboration, Measurement of the Lund Jet Plane Using Charged Particles in 13 TeV Proton-Proton Collisions with the ATLAS Detector, Phys. Rev. Lett. 124 (2020) 222002, 2004.03540 [hep-ex].

[37] ATLAS collaboration, Measurement of the Lund jet plane using charged particles in $13 \mathrm{TeV}$ proton-proton collisions with the ATLAS detector, 2020.

https://doi.org/10.17182/hepdata.93183.

[38] A. Lifson, G. P. Salam and G. Soyez, Calculating the primary Lund Jet Plane density, 2007.06578 [hep-ph]. 